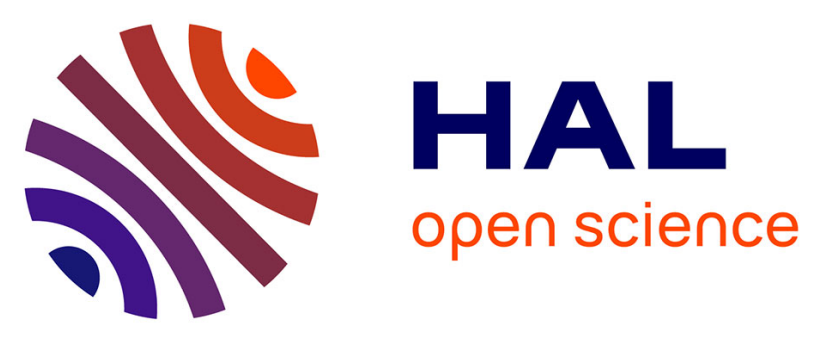

\title{
EPR and electrical conductivity of the organic superconductor di-tetramethyltetraselenafulvalenium-perchlorate, (TMTSF)2ClO4 and a metastable magnetic state obtained by fast cooling
}

\author{
S. Tomić, D. Jérome, P. Monod, K. Bechgaard
}

\section{To cite this version:}

S. Tomić, D. Jérome, P. Monod, K. Bechgaard. EPR and electrical conductivity of the organic superconductor di-tetramethyltetraselenafulvalenium-perchlorate, (TMTSF)2ClO4 and a metastable magnetic state obtained by fast cooling. Journal de Physique Lettres, 1982, 43 (23), pp.839-844. 10.1051/jphyslet:019820043023083900 . jpa-00232133

HAL Id: jpa-00232133

https://hal.science/jpa-00232133

Submitted on 1 Jan 1982

HAL is a multi-disciplinary open access archive for the deposit and dissemination of scientific research documents, whether they are published or not. The documents may come from teaching and research institutions in France or abroad, or from public or private research centers.
L'archive ouverte pluridisciplinaire HAL, est destinée au dépôt et à la diffusion de documents scientifiques de niveau recherche, publiés ou non, émanant des établissements d'enseignement et de recherche français ou étrangers, des laboratoires publics ou privés. 


\title{
EPR and electrical conductivity of the organic superconductor di-tetramethyltetraselenafulvalenium-perchlorate, (TMTSF) ${ }_{2} \mathrm{ClO}_{4}$ and a metastable magnetic state obtained by fast cooling
}

\author{
S. Tomić $(*)$, D. Jẹrome, P. Monod and K. Bechgaard (**) \\ Laboratoire de Physique des Solides, Bât. 510, Université de Paris-Sud, 91405 Orsay, France \\ (Reçu le 20 juillet 1982, révisé le 21 septembre, accepté le 8 octobre 1982)
}

\begin{abstract}
Résumé. - Les mesures de la conductioñ électrique et de la RPE dans (TMTSF) ${ }_{2} \mathrm{ClO}_{4}$ effectuées après un cyclage contrôlé de la température démontrent l'existence d'une transition de phase vers $22 \mathrm{~K}$. Un refroidissement lent $(12 \mathrm{~K} / \mathrm{h})$ conduit à un état présentant un paramagnétisme de Pauli et de la supraconduction respectivement au-dessus et au-dessous de $1,2 \mathrm{~K}$. Au contraire un refroidissement rapide $(30 \mathrm{~K} / \mathrm{min}$.) conduit à un état magnétique (SDW) métastable au-dessous de $4 \mathrm{~K}$ comme l'indiquent une augmentation de la résistivité et la disparition du signal RPE à basse température.
\end{abstract}

\begin{abstract}
EPR and electrical conductivity measurements under controlled thermal cycles demonstrate that a phase transition is occurring at about $22 \mathrm{~K}$ in (TMTSF) ${ }_{2} \mathrm{ClO}_{4}$. Slow cooling $(12 \mathrm{~K} / \mathrm{h})$ yields a stable ground state exhibiting Pauli paramagnetism above and superconductivity below $1.2 \mathrm{~K}$. In contrast, fast cooling $(30 \mathrm{~K} / \mathrm{min}$.) yields a metastable magnetic (SDW) state below $4 \mathrm{~K}$ as indicated by increasing resistivity and disappearance of the EPR signal with decreasing temperature.
\end{abstract}

1. Introduction. - (TMTSF) ${ }_{2} \mathrm{ClO}_{4}$ is so far the only member of the family of (TMTSF) ${ }_{2} \mathrm{X}$ salts where a superconducting (SC) ground state is observed at ambient pressure [1]. Specific heat measurements showed that the actual transition temperature depends on the cooling rate decreasing from $1.2 \mathrm{~K}$ to $0.9 \mathrm{~K}$ under rapid cooling conditions [2]. NMR investigation [3] clearly demonstrated that the rapid cooling process establishes a magnetic (SDW) state below $4 \mathrm{~K}$ for magnetic fields between $10 \mathrm{kOe}$ and $.65 \mathrm{kOe}$, under the same conditions as when the $\mathrm{SC}$ transition temperature is depressed to $0.9 \mathrm{~K}$. It was further suggested that this is related to disorder in the anion lattice frozen in by rapid cooling.

We report additional characterization by EPR (at $3.2 \mathrm{kOe}$ ) and by resistivity measurements of the two states obtained by either rapid (quenched-Q) or slow cooling (relaxed-R), identify the important temperature region for the transition, and estimate the activation energy involved.

(*) Permanent address : Institute of the Physics of the University, P.O.B. 304, 41001 Zagreb, Yugoslavia.

(**) Permanent address : H. C. Oersted Institute, Universitetsparken 5, DK-2100, Copenhagen, Denmark. 
2. Experimental and results. - All EPR runs were done on one single crystal of (TMTSF) ${ }_{2} \mathrm{ClO}_{4}$, $5 \mathrm{~mm}$ long and with a cross section of $0.33 \times 0.11 \mathrm{~mm}^{2}$. The crystal was mounted on a plexiglass holder with a small drop of silicon grease on one end of the crystal to avoid strain during the cooling process as much as possible. The static magnetic field $\left(H_{0}\right)$ is perpendicular to the needle axis and the crystal can be rotated in the $b^{\prime} c^{*}$ plane [4]. A minimum loss was obtained with $H_{0}$ parallel to $c^{*}$ where all reported results were obtained. The experiments were performed at 9.4 GHz using a standard EPR spectrometer. Cooling and temperature control were obtained using an Oxford Instruments continuous-flow helium system and a temperature controller with an iron : gold/chromel thermocouple. The maximum power which can be delivered to the sample cavity is of the order of $100 \mathrm{~mW}$. Normal operating level was $-6 \mathrm{~dB}$ from the maximum and care was taken to avoid saturation and heating.

The cooling rate experiments were performed as defined below. The recorded field derivative of the absorption EPR signal is characterized by the following three quantities : the intensity $(I)$ for symmetric (to within the noise, Lorentzian) and asymmetric lines [5], the linewidth ( $L W$ ) and the ratio of peak amplitudes $(B / A)$, to describe the asymmetry of the lineshape (Fig. 1). The

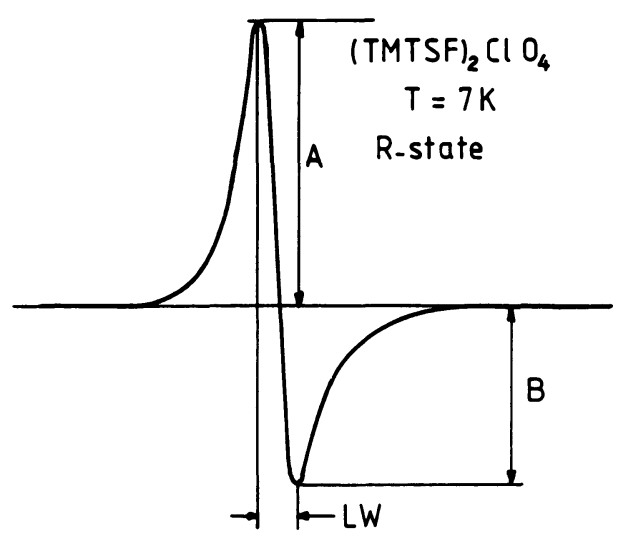

Fig. 1. - EPR signal in (TMTSF) ${ }_{2} \mathrm{ClO}_{4}$ at $7 \mathrm{~K} . A$ and $B$ are the peak amplitudes in lower and higher field, respectively. The linewidth $L W$ is the peak to peak width without correction for $B / A$.

line intensity for both lines was calculated as $I=(A+B) \times L W^{2} . A$ and $B$ are the peak amplitudes in the lower and higher field region, respectively. $L W$ is the peak to peak width. Results are shown in figure 2 .

The thermal treatment variations were as follows :

(a) (Relaxed state - R). The sample was cooled from $40 \mathrm{~K}$ at a rate of $12 \mathrm{deg}$. $/ \mathrm{h}$. The signal was monitored as the sample cooled. No difference was observed when cooling from higher $T$ or even slower.

(b) (Quenched state - Q). Cooling from $40 \mathrm{~K}$ at a rate of $30 \mathrm{deg} . / \mathrm{min}$. to $3.5 \mathrm{~K}$. The signal was monitored when heating rather quickly to avoid annealing $(c)$, which starts to be important around $18 \mathrm{~K}$.

(c) (Annealing process). The sample is quenched $(b)$ and the temperature raised quickly ( $<30 \mathrm{~s}$ ) to the annealing temperature $\left(T_{\text {ann }}\right)$. After resting at $T_{\text {ann }}$ for a fixed time $\Delta t$, the sample is quickly cooled again to $3.5 \mathrm{~K}$ and the signal recorded. This enables an estimate of the thermo- 
R-STATE Q-STATE

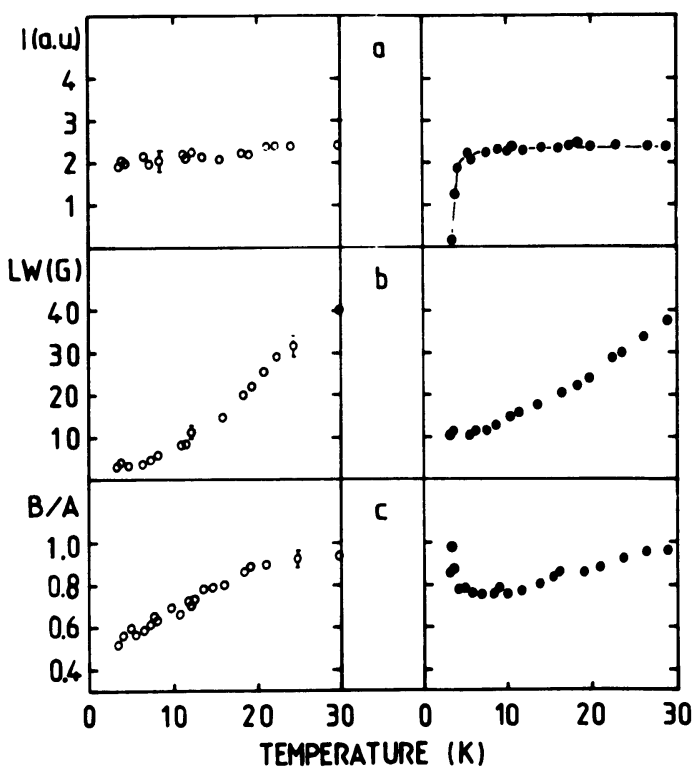

Fig. 2. - EPR results obtained in slow cooling (open circles $\mathrm{R}$-state) and in rapid cooling (solid circles $\mathrm{Q}$ state, data taken in warming). a) The line intensity $I=(A+B) \times L W^{2}$ in arbitrary units versus temperature $T$. The solid line is a guide for the eye. $b$ ) The linewidth $L W$ in gauss versus temperature $T . c$ ) The ratio of amplitudes $B / A$ versus temperature $T$.



Fig. 3. - Normalized resistance versus temperature. Open circles for slow cooling and solid circles for rapid cooling (data taken in warming). A partly annealed sample obtained by cooling from $30 \mathrm{~K}$ in $15 \mathrm{~min}$. is shown as triangles. The beginning of the SC transition is seen at $1.2 \mathrm{~K}$. The solid line is a guide for the eye. 
dynamics of the phase transition occurring at about $22 \mathrm{~K}$. In this way the temperature region between 10-30 $\mathrm{K}$ was explored.

Figure 4 shows the normalized intensity $I / I_{\mathrm{R}}$, linewidth $L W / L W_{\mathrm{Q}}$ and lineshape $\left(\frac{B}{A}\right) /\left(\frac{B}{A}\right)_{\mathrm{Q}}$ at $3.5 \mathrm{~K}$ versus the annealing temperature $T_{\text {ann }}$ for two different annealing times $\Delta t$. $I_{\mathrm{R}}$ is the intensity in the $\mathrm{R}$-state, $L W_{\mathrm{Q}}$ is the linewidth in the $\mathrm{Q}$-state and $\left(\frac{B}{A}\right)_{\mathrm{Q}}$ is the peak amplitude ratio in the Q-state, at $3.5 \mathrm{~K}$.

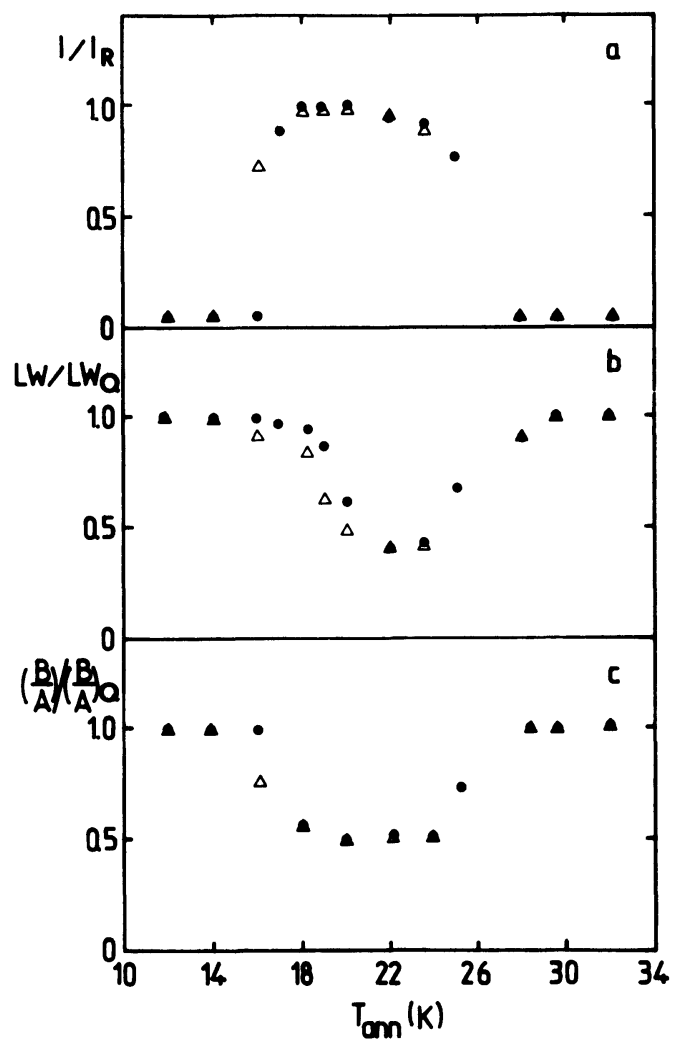

Fig. 4. - Normalized intensity $I / I_{\mathrm{R}}(a)$, linewidth $L W / L W_{\mathrm{Q}}(b)$ and peak amplitude ratio $\left(\frac{B}{A}\right) /\left(\frac{B}{A}\right)_{\mathrm{Q}}(c)$ at $3.5 \mathrm{~K}$ versus the annealing temperature $T_{\text {ann }}$, for two different annealing times $\Delta t$. Circles for $\Delta t=3 \mathrm{~min}$, triangles for $\Delta t=13 \mathrm{~min} . I_{\mathrm{R}}$ is the intensity in the R-state. $L W_{\mathrm{Q}}$ is the linewidth in the Q-state. $\left(\frac{B}{A}\right)_{\mathrm{Q}}$ is the peak amplitude ratio in the Q-state.

For the shortest experimental annealing time $\Delta t=3 \mathrm{~min}$., the effective annealing temperature region lies between $18-24 \mathrm{~K}$. There is an almost complete temperature coincidence in the maximum and minimum of the intensity and lineshape curves respectively, whereas a minimum in the linewidth curve is more narrow and shifted towards higher temperatures. We define the transition temperature to the ordered state as the temperature when all three quantities recover their relaxed values. Thus, we get $T \sim 22 \mathrm{~K}$. 
Electrical conductivity experiments were performed at ambient pressure using a low frequency $(70 \mathrm{~Hz})$ ac method with a measuring current of $10 \mu \mathrm{A}$. Four electrical contacts were made with silver paint on previously evaporated gold pads. Resistivity measurements were all performed using two samples of approximate dimensions $4 \times 1 \times 0.05 \mathrm{~mm}^{3}$. During cooling down from room temperature microcracks give rise to resistivity jumps of different magnitude, but the temperature behaviour below $50 \mathrm{~K}$ is in all runs qualitatively the same for both samples. The behaviour of the sample presented in figure 3 was qualitatively and quantitatively reproducible in several runs.

Thermal treatment similar to $(a)$ and $(b)$ was performed. It was possible to quench faster ( $\sim 50 \mathrm{deg} . / \mathrm{min}$.) and again record resistivity on warming. Experiments were also performed to check the annealing effect. The resistivity measurements displayed in figure 3 were performed as follows : the sample was first cooled slowly down (shown as open circles, $\sim 12 \mathrm{~K} / \mathrm{h}$ ). It was rewarmed to $\sim 40 \mathrm{~K}$ and then cooled rapidly down (shown as solid circles, $\sim 50 \mathrm{~K} / \mathrm{min}$.). Finally the sample was rewarmed once again to anneal and " relaxed » by slow cooling down (shown as triangles, $\sim 80 \mathrm{~K} / \mathrm{h}$ ).

3. Discussion. - The striking result of these experiments is as follows. The quenching process results in a reduction of $\chi_{\text {spin }}$ below about $4 \mathrm{~K}$. According to the NMR results [3] the quenched SDW state is stable at low temperatures. Under quenching conditions the resistivity increases abruptly below $4 \mathrm{~K}$. This strongly indicates the severe influence of the quenching process on the interplay between SDW and SC. Furthermore we observe that the slowly cooled R-state exhibits Pauli type paramagnetism and continuously decreasing resistivity in the same temperature region. An anomaly at $25 \mathrm{~K}$ was already noticed by accurate resistivity experiments [6] and suggested to be the signature of a phase transition. We establish a transition temperature around $22 \mathrm{~K}$ and our experiment allows a rough estimate of the activation energy for the transition of about $300 \mathrm{~K}$ (see below). The differences in the response of the EPR signal were already noticed [7], but not evaluated and not connected with the varying resistance at low temperature.

The Q-state is magnetic below $4 \mathrm{~K}$ or so and simultaneously the resistivity increases. The features resemble closely the features observed in other (TMTSF) ${ }_{2} \mathrm{X}$ salts when the SDW condenses (see e.g. ref. [5]). Furthermore, Walsh et al. [8] have observed AFMR (probably) under quenching conditions and the NMR measurements [3] clearly show the onset of strong local fields below $4 \mathrm{~K}$ in the Q-state. The present measurements in lower field $(3.2 \mathrm{kOe})$ extend the proposed phase diagram.

The R-state is characterized by a Pauli-like susceptibility $I_{R}$ and constantly increasing electrical conductivity $\sigma_{\mathrm{R}}$. The linewidth is narrow $L W_{\mathrm{R}} \sim 3$ gauss and the line is asymmetric. In order to explore quantitatively this feature of the EPR lineshape a full analysis of the corresponding geometry within Dyson's theory is needed. We do observe a qualitative agreement between the $B / A$ (Fig. $2 c$ ) and the resistivity (Fig. 3) temperature behaviour. In the Q-state as the resistivity increases the $B / A$ ratio goes towards unity, whereas in the R-state with decreasing resistivity the line is asymmetric (Fig. $2 c$ ) down to the lowest measured temperatures. An accurate analysis is however further complicated by skin depth effects $[9,10]$ because the skin depth is comparable with the given crystal cross section below about $15 \mathrm{~K}$.

Annealing process - Annealing as manifested in the resistivity behaviour is demonstrated in figure 3. Cooling from $30 \mathrm{~K}$ to $4.2 \mathrm{~K}$ during $15 \mathrm{~min}$. (intermediate rate, triangles in Fig. 3) leads to a partially relaxed state with only a mild upturn at low temperature. Similarly, a partly relaxed state shows intermediate EPR behaviour (smaller and broader signal). The EPR results of the annealing experiments (shown in Fig. 4) indicate a transition temperature of about $22 \mathrm{~K}$, since rapid cool down from higher temperature gives parameters characteristic of the Q-state.

The result obtained for annealing temperatures below $22 \mathrm{~K}$ clearly indicates an activated nature of the change towards the R-state. Defining $\tau_{1 / 2 \text { ann }}$ as the time after which at a given 
temperature, $T_{\text {ann }}$, the parameters recover half the value of the R-state, we use the expression $\tau_{1 / 2 \text { ann }} \simeq \tau_{x} \mathrm{e}^{\varepsilon_{a} / T_{\mathrm{ann}}}$ to get a crude estimate of the activation energy. For $T=16,17$ and $18 \mathrm{~K}$ we find $\tau_{1 / 2 \text { ann }} \simeq 9,1.65$ and $1.5 \mathrm{~min}$. respectively and obtain an $\varepsilon_{a}$ of about $300 \mathrm{~K}$.

The authors of reference [3] have proposed a model for the quenched state in terms of lattice disorder. At high temperatures the $\mathrm{ClO}_{4}$ anions randomly occupy two equivalent positions [4], whereas at low temperatures the anions are suggested to display a ferroelectric type of ordering with no change in the lattice periodicity. The quenching is suggested to freeze in disorder. This interpretation is recently supported by X-ray investigation [11], which shows the existence of a phase transition at $24 \mathrm{~K}$ with precursors below $30 \mathrm{~K}$, giving rise to the periodicity $(a, 2 b, c)$. The effect of the anion disorder in the Q-state was suggested [3] to lead to depression of the SC instability. This in turn should allow the competing SDW instability.

The results of Garoche et al. indicated a SC transition at $0.9 \mathrm{~K}$ (vs. $1.2 \mathrm{~K}$ in the R-state) under their quenching conditions $(10 \mathrm{~K} / \mathrm{min}$.). We emphasize that this corresponds to our " intermediate " result. Preliminary experiments down to milli $\mathrm{K}$ temperatures indicate the SC transition can possibly be suppressed under quenching conditions and we also notice that in the temperature region 4-20 $\mathrm{K}$ quenching gives rise to additional scattering.

In conclusion, we have shown that slow cooling below $30 \mathrm{~K}$ in (TMTSF) ${ }_{2} \mathrm{ClO}_{4}$ allows a full phase transformation at about $22 \mathrm{~K}$ leading to a low temperature ground state (relaxed R-state) with enhanced conductivity and a finite Pauli-like susceptibility above $3.5 \mathrm{~K}$. In contrast rapid cooling below $30 \mathrm{~K}$ leads to a low temperature metastable state (quenched Q-state) with increased resistivity and vanishing EPR susceptibility. This state may be identified as the state showing SDW character [8]. The annealing process in the temperature region $15-30 \mathrm{~K}$ recovers the $\mathrm{R}$-state proportional to the annealing time and annealing temperature, thus leading to a mixed state.

As a consequence of the present work it is tempting to suggest that the structural transition at ambient pressure at $180 \mathrm{~K}$ leading to a superstructure $(2 a, 2 b, 2 c)$ in $(\mathrm{TMTSF})_{2} \operatorname{ReO}_{4}[12,13]$ (antiferroelectric type order) is transformed under pressure into a ferroelectrically ordered phase $(a, 2 b, c)$ as found in (TMTSF) ${ }_{2} \mathrm{ClO}_{4}$. Thus, the intermediate pressure phase in (TMTSF) ${ }_{2} \operatorname{ReO}_{4}$ [13] with large dependent resistive anomaly (hysteresis effect) may be a metastable mixture of the two phases.

Acknowledgments. - We thank T. Takahashi and R. Brusetti for fruitful discussions and A. Andrieux for skillful technical assistance.

\section{References}

[1] Bechgaard, K., Carneiro, K., Olsen, M., Rasmussen, F. B. and Jacobsen, C. S., Phys. Rev. Lett. 46 (1981) 852.

[2] Garoche, P., Brusetti, R. and Bechgaard, K., submitted to Phys. Rev. Lett.

[3] Takahashi, T., Jérome, D. and Bechgaard, K., J. Physique-Lett. 43 (1982) L-565.

[4] Bechgaard, K., Carneiro, K., Rasmussen, F. B., Olsen, M., Rindorf, G., Jacobsen, C. S., PederSEN, H. J. and SCOTT, J. C., J. Am. Chem. Soc. 103 (1981) 2440.

[5] Pedersen, H. J., Scott, J. C. and Bechgaard, K., Phys. Rev. B 24 (1981) 5014.

[6] Gubser, D. U., Fuller, W. W., Poehler, T. O., Stokes, J., Cowan, D. O., Lee, M. and Bloch, A. N., Mol. Cryst. Liq. Cryst. 79 (1982) 225.

[7] ScotT, J. C., Mol. Cryst. Liq. Cryst. 79 (1982) 49.

[8] Walsh Jr., W. M., Bull. Am. Phys. Soc. 27 (1982) 150 and preprint.

[9] Pifer, J. H. and Magno, R., Phys. Rev. B (1971) 663.

[10] Feher, G. and KIP, A. F., Phys. Rev. 98 (1955) 337.

[11] Pouget, J. P., Shirane, G., Bechgaard, K. and Fabre, J. P., submitted to Phys. Rev. Lett.

[12] Jacobsen, C. S., Pedersen, H. J., Mortensen, K., Rindorf, G., Thorup, N., Torrance, J. B. and BechgaARD, K., J. Phys. C 15 (1982) 2651.

[13] Parkin, S. S. P., Jérome, D. and Bechgaard, K., Mol. Cryst. Liq. Cryst. 79 (1982) 213. 